\title{
Reemergence of porcine epidemic diarrhea virus on Jeju Island
}

\author{
Sunhee Lee ${ }^{1}$, Deok-Ho Ko ${ }^{2}$, Seong-Kyu Kwak ${ }^{2}$, Chung-Hun Lim² ${ }^{2}$, Sung-Up Moon ${ }^{3}$, Du Sik Lee ${ }^{4}$, Changhee Lee ${ }^{1, *}$ \\ ${ }^{1}$ Animal Virology Laboratory, School of Life Sciences, BK21 Plus KNU Creative BioResearch Group, \\ Kyungpook National University, Daegu 702-701, Korea \\ ${ }^{2}$ Hanbat Swine Veterinary Service, Jeju 695-932, Korea \\ ${ }^{3}$ Veterinary Research Institute, Jeju Special Self-Governing Province, Jeju 690-700, Korea \\ ${ }^{4}$ College of Veterinary Medicine, Jeju National University, Jeju 690-756, Korea
}

(Received: April 25, 2014; Accepted: June 18, 2014)

\begin{abstract}
Porcine epidemic diarrhea virus (PEDV) strains responsible for recent outbreaks in the United States have been occurring in Mainland Korea since late 2013. Over the past 10 years, PEDV outbreaks have not been reported on Jeju Island. However, in late March of 2014, PEDV re-emerged on Jeju Island and was found to be genetically identical to PEDV strains currently circulating in Mainland Korea. The present study was conducted to provide a better understanding of the epidemiology of PEDV and more effective preventive measures against PED.
\end{abstract}

Keywords : Jeju Island, Korea, porcine epidemic diarrhea virus, reemergence, US strain-like strains

Porcine epidemic diarrhea (PED) is a devastating pig enteric disease causing lethal watery diarrhea followed by severe dehydration with high mortality in neonatal piglets [10]. The disease was first recognized in England in 1971 [7], and since then, outbreaks have been reported in Europe, Asia, and recently, North America including the United States [1, $9,13,14]$. The causative agent of this disease, the PED virus (PEDV), belongs to the Alphacoronavirus genus within the family Coronaviridae of the order Nidovirales [8, 10]. The spike (S) protein of PEDV is the major virion envelope glycoprotein; interacts with the host cellular receptor for virus entry and mediates neutralizing antibodies. Therefore, the PEDV $\mathrm{S}$ protein is an appropriate viral gene for sequencing and molecular analyses to investigate the genetic relatedness of PEDV isolates and the epidemiological status of PEDV in the field [4].

The first case of PED in Korea was confirmed in 1992 [3] and since its emergence, PED outbreaks have continuously occurred every year, leading to a serious production loss to the Korean pork industry up until early 2010 . However, after severe outbreaks of foot-and-mouth disease (FMD) in 2010 and 2011, the prevalence of PEDV infections in Korea was significantly reduced with only sporadic outbreaks. In November of 2013, PEDV suddenly recurred and subsequently, has swept rapidly across Mainland Korea. Genetic and phylogenetic analyses revealed that PEDV isolates responsible for the recurrence in Mainland Korea are most highly related to PEDV strains currently circulating in the United States [5]. The last PEDV outbreak on Jeju Island was reported in 2004 and since then, PEDV has not emerged again on Jeju Island in almost 10 years. Beginning in late March of 2014, PEDV re-emerged in the Hanlim area of Jeju Island and subsequently spread to other districts, resulting in high mortality in newborn piglets. To determine the origin and diversity of PEDV responsible for the ongoing outbreaks on Jeju Island, we sought to determine the full-length sequences of the $S$ proteins of field isolates identified from affected pig farms.

From late March 2014 through early April 2014, 5 stool specimens were taken from sows and piglets with acute watery diarrhea at different swine farms. Fecal samples were processed into $10 \%$ suspensions as described previously [4] and initially subjected to RT-PCR using a TGE/PED Detection Kit (iNtRON Biotechnology, Korea) according to the manufacturer's protocol. The PEDV-positive supernatants were stored at $-80^{\circ} \mathrm{C}$ as the field isolate until use.

In order to sequence the complete $\mathrm{S}$ glycoprotein gene of the re-emergent Jeju PEDV isolates, two overlapping cDNA fragments spanning the entire $S$ gene were RT-PCR amplified as described previously [4]. The individual cDNA amplicons were gel-purified, cloned into pGEM-T Easy (Promega, USA) and sequenced in both directions using two commercial vector-specific T7 and SP6 primers and the S gene-specific primers. General procedures for DNA manipulation and cloning were performed according to standard procedures [12]. The

*Corresponding author

Tel: $+82-53-950-7365$, Fax: $+82-53-955-5522$

E-mail: changhee@knu.ac.kr 


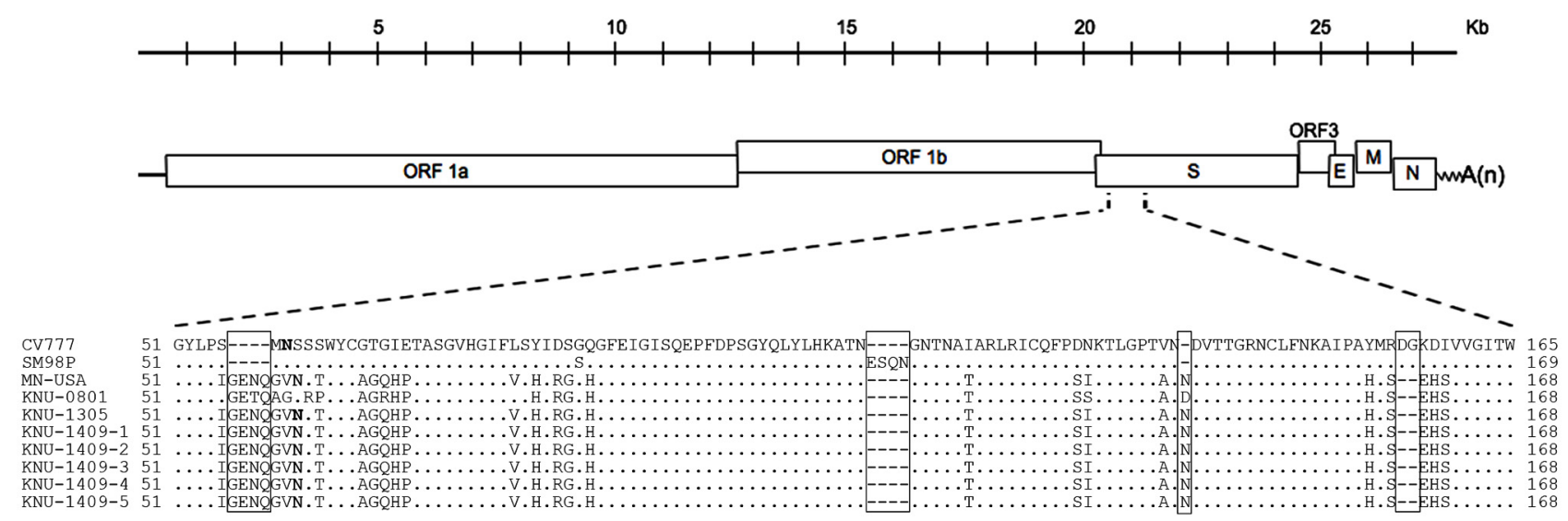

Fig. 1. Amino acid sequence alignment of the N-terminal region of the spike (S) protein between the reference strains and 5 reemergent Jeju strains. The top illustration represents the organization of the Porcine epidemic diarrhea virus (PEDV) genome, and only the corresponding alignment of amino acid sequences of the N-terminal region containing deletions is shown. The dashes (-) indicate the deleted sequences. Potential N glycosylation sites predicted by GlycoMod Tool are shown in boldface type. Insertions and deletions within PEDV isolates are indicated by solid boxes.

full-length $\mathrm{S}$ glycoprotein gene sequences of the re-emergent Jeju PEDV isolates were deposited to the GenBank database under their accession numbers (KJ741221 to KJ741225).

The sequences of 33 fully sequenced $\mathrm{S}$ genes of PEDV isolates were used in sequence alignments and phylogenetic analyses. The multiple-sequencing alignments were generated with the ClustalX 2.0 program [16], and the percentages of nucleotide sequence divergences were further assessed with the same software program. Phylogenetic trees were constructed from the aligned nucleotide or amino acid sequences by using the neighbor-joining method and subsequently subjected to bootstrap analysis with 1,000 replicates in order to determine percentage reliability values on each internal node of the tree [11]. All tree figures were produced using Mega 4.0 software [15].

The complete nucleotide sequences of the $\mathrm{S}$ genes of all PEDV strains isolated recently on Jeju Island were genetically characterized. The sequence data indicated that the $\mathrm{S}$ genes of all re-emergent Jeju PEDV isolates (KNU-1409-1 to 1409-5) consisted of 4,161 nucleotides (nt), which encoded 1,386 amino acid (aa) residues. The full-length $\mathrm{S}$ genes of the recent Jeju PEDV strains were determined to be 9-nt ( 3 aa) longer than the prototype PEDV strain, CV777. This correlated with the presence of genetic signatures for recent PEDV field isolates that involve 2 notable 4-aa and 1-aa insertions at positions 55 and 56 and positions 135 and 136, respectively, and a unique 2-aa deletion located between positions 160 and 161, compared to CV777 (Fig. 1), which have been identified previously in all PEDV strains isolated in Korea, China, and the United States [2, 4, 6].

The similarity between the $\mathrm{S}$ protein genes was measured. The sequence results showed high homology between the 5 Jeju PEDV isolates, ranging from 99.8 to $99.9 \%$ identity. Nucleotide sequence analysis was further extended to the deduced amino acid level and demonstrated 99.9 to $100 \%$ identity between the isolates. In addition, all re-emergent Jeju isolates had diverse similarities with the previous Korean field isolate (KNU-0801), showing 94.5 to $94.6 \%$ homology at the nucleotide sequence level and, accordingly, the amino acid identity ranged between 94.2 and $94.3 \%$. However, when the 5 isolates were compared with the recent Korean and US PEDV strains, they were almost identical and were consistently within the range of from 99.0 to $99.8 \%$ and 99.0 to $99.8 \%$ identities at the nucleotide and amino acid levels, respectively. Thus, our data reveal that the re-emergent Jeju PEDV isolates were highly homologous with the strains responsible for the recent outbreaks in Mainland Korea and the United States.

Since the full-length S gene and the S1 portion (1-735 aa) are known to be suitable regions for analyzing genetic relatedness among different PEDV isolates [4], we performed phylogenetic analyses using both regions to establish the genetic relationships of the Jeju PEDV strains. The full-length $S$ gene-based phylogenetic tree revealed that the PEDV strains were divided into 2 separate clusters, designated as genogroup 1 (G1) and genogroup 2 (G2); G2 then consisted of at least 2 subgroups, designated as $2 \mathrm{a}$ and $2 \mathrm{~b}$ (Fig. 2). As shown in Fig. 2A, all of the re-emergent Jeju PEDV strains were classified into subgroup $2 \mathrm{~b}$ along with the current Mainland Korean isolates, which were most closely clustered together with the emergent US strains in an adjacent clade with the same subgroup. The data suggest that the US strain-like Korean strain is the origin of the re-emergence of PEDV on Jeju Island. In addition, subsequent phylogenetic analysis of the $\mathrm{S} 1$ portion showed the same cluster structure as the phylogenetic tree based on the full-length $\mathrm{S}$ gene (Fig. 2B).

Sequence comparison and phylogenetic analyses indicated that the re-emergent Jeju PEDV isolates were most genetically similar to PEDV strains that are currently emerging in Mainland Korea and the United States in 2013. Therefore, 
A

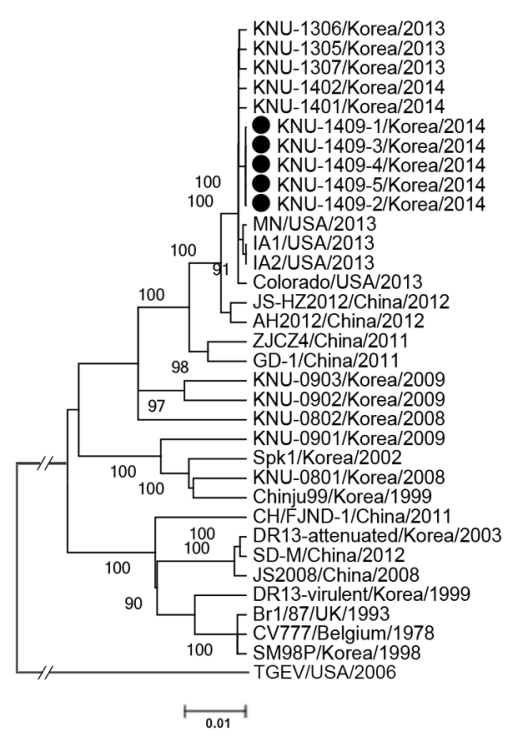

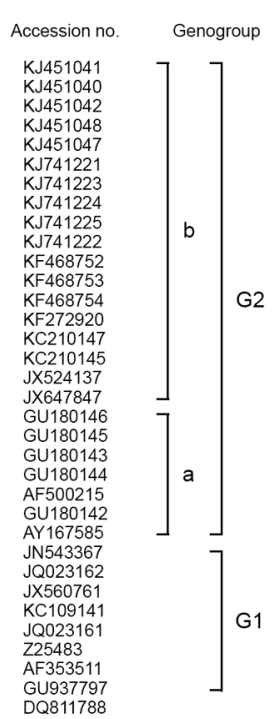

B

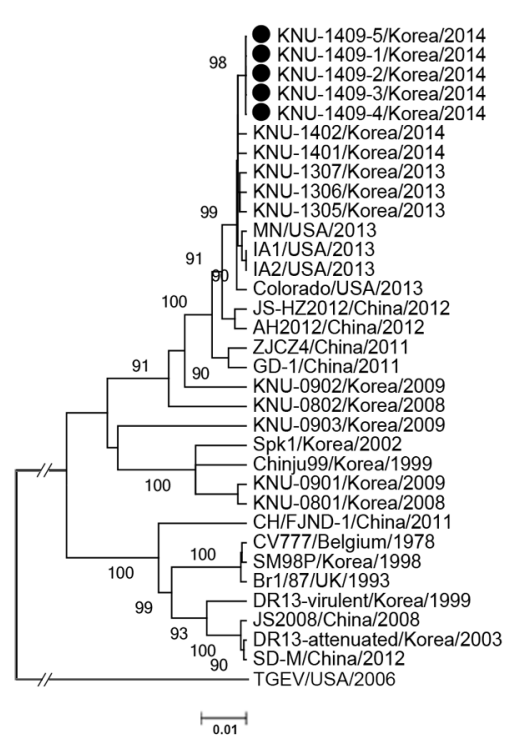

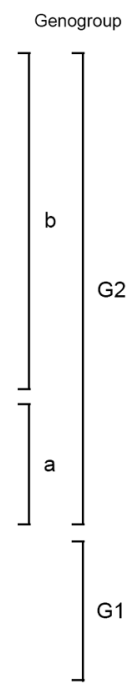

Fig. 2. Phylogenetic analyses based on the nucleotide sequences of the full-length $S$ gene (A) and S1 portion (B) of PEDV strains. A putative similar region of the S protein of transmissible gastroenteritis virus (TGEV) was included as an outgroup in this study. Multiple-sequencing alignments were performed using the ClustalX program, and the phylogenetic tree was constructed from the aligned nucleotide sequences by using the neighbor-joining method. The numbers at each branch represent bootstrap values greater than $50 \%$ of 1,000 replicates. The scale bars indicate 0.01 inferred substitutions per site. The names of the strains, countries, and years of isolation, GenBank accession numbers, and genogroups and subgroups proposed in this study are shown. Solid circles indicate the 5 reemergent Jeju strains that are similar to the recent Korean and US PEDV strains.

our results indicate that the recent re-emergent Jeju strains originated directly from Mainland Korea. PEDV is mainly spread via the fecal-oral route, and the most common sources of infected feces are pigs, trucks, boots, clothing, or other fomites. Therefore, PEDV can be newly introduced into pig farms directly via infected piglets or gilts, or indirectly, either by trailers transporting pigs, manure, or feed, by people, including visitors such as swine practitioners or truck drivers, or via other sources such as wild animals or birds. Considering these factors, we hypothesized several scenarios to explain how the recent US strain-like PEDV strains could have been introduced onto Jeju Island from Mainland Korea. In the case of PEDV outbreaks on Jeju Island, pigs may not be the source for PEDV transmission, because purchasing breeding stock from Mainland Korea has been restricted since 2000. The most important potential source for PEDV spread to Jeju Island could be vehicles. Indeed, during recent PEDV outbreaks in Mainland Korea, some trailers have delivered high-parity sows to slaughter plants located in Mainland Korea, but the vehicles and their drivers might not be thoroughly disinfected before re-entering Jeju Island. Additionally, we were unable to exclude the possibility of transmission via foreigners from Mainland Korea visiting pig farms on Jeju Island, even though they seem to be one of the least possible sources.

In 2010 to 2011, severe outbreaks of foot-and-mouth disease (FMD) occurred across the country causing the mass cull of over $30 \%$ (3 million) of the entire domestic pig population in Korea. However, the 2010-2011 FMD outbreaks affected only Mainland Korea and did not reach Jeju Island, which was likely due to reinforced biosecurity and control that strictly blocked possible sources and routes, including animals, vehicles, and people. The Jeju provincial government made these preventive measures mandatory, because FMD is considered as a class I communicable animal disease in Korea, and it is a World Organization for Animal Health (OIE) reportable disease, which affects export markets. In contrast, PED is considered as a class III communicable animal disease in Korea and is not an OIE reportable disease. Thus, despite the rampant spread of PEDV in Mainland Korea, the Jeju provincial government might not have reacted swiftly and applied the disease control protocols necessary based upon the class III communicable animal disease classification. PEDV is a highly epizootic virus that causes $90-100 \%$ mortality in piglets less than 1-week-old and spreads quickly to other farms and areas, which leads to tremendous financial impacts for both pork producers and meat consumers. The Jeju provincial government should adequately reinforce the current quarantine and control procedures to pay particular attention to anything or anyone sourced for disease transmission while a deadly virus, such as PEDV is emerging in Mainland Korea. In addition, a molecular epidemiology study is further needed to provide temporal and geographical evidence for the exact transmission 
route of the recent re-emergent PEDV strains on Jeju Island. To prevent the periodic recurrence of acute PEDV outbreaks, a proper vaccination program should be initiated to enhance overall immunity to PEDV in all stock along with strict biosecurity. The present study provides a better understanding of the importance of taking effective preventive measures and biosecurity against PED to prevent spread of the disease.

\section{Acknowledgments}

This research was supported by Technology Development Program for Bio-industry, Ministry for Agriculture, Food and Rural Affairs, Korea (311007-05-1-HD120).

\section{References}

1. Chen JF, Sun DB, Wang CB, Shi HY, Cui XC, Liu SW, Qiu HJ, Feng L. Molecular characterization and phylogenetic analysis of membrane protein genes of porcine epidemic diarrhea virus isolates in China. Virus Genes 2008, 36, 355-364.

2. Huang YW, Dickerman AW, Piñeyro P, Li L, Fang L, Kiehne R, Opriessnig T, Meng XJ. Origin, evolution, and genotyping of emergent porcine epidemic diarrhea virus strains in the United States. MBio 2013, 4, e00737-13.

3. Kweon CH, Kwon BJ, Jung TS, Kee YJ, Hur DH, Hwang EK, Rhee JC, An SH. Isolation of porcine epidemic diarrhea virus (PEDV) in Korea. Korean J Vet Res 1993, 33, 249254.

4. Lee DK, Park CK, Kim SH, Lee C. Heterogeneity in spike protein genes of porcine epidemic diarrhea viruses isolated in Korea. Virus Res 2010, 149, 175-182.

5. Lee $\mathbf{S}$, Lee $\mathbf{C}$. Outbreak-related porcine epidemic diarrhea virus strains similar to US strains, South Korea, 2013. Emerg Infect Dis 2014. 20, 1223-1226.

6. Li W, Li H, Liu Y, Pan Y, Deng F, Song Y, Tang X, He Q. New variants of porcine epidemic diarrhea virus, China, 2011. Emerg Infect Dis 2012, 18, 1350-1353.
7. Oldham J. Letter to the editor. Pig Farming 1972, 10, 7273.

8. Pensaert MB, de Bouck P. A new coronavirus-like particle associated with diarrhea in swine. Arch Virol 1978, 58, 243-247.

9. Puranaveja S, Poolperm P, Lertwatcharasarakul P, Kesdaengsakonwut $\mathbf{S}$, Boonsoongnern A, Urairong $\mathbf{K}$, Kitikoon P, Choojai P, Kedkovid R, Teankum K, Thanawongnuwech R. Chinese-like strain of porcine epidemic diarrhea virus, Thailand. Emerg Infect Dis 2009, 15, 11121115.

10. Saif LJ, Pensaert MB, Sestack K, Yeo SG, Jung K. Coronaviruses. In: Zimmerman JJ, Karriker LA, Ramirez A, Schwartz KJ, Stevenson GW (eds.). Diseases of Swine. 10th ed. pp. 501-524. Wiley-Blackwell, Ames, 2012.

11. Saitou N, Nei M. The neighbor-joining method: a new method for reconstructing phylogenetic trees. Mol Biol Evol 1987, 4, 406-425.

12. Sambrook JF, Russell DW. Molecular Cloning: A Laboratory Manual, 3rd ed. pp. 1.1-1.31, Cold Spring Harbor Laboratory Press, New York, 2001.

13. Stevenson GW, Hoang H, Schwartz KJ, Burrough ER, Sun D, Madson D, Cooper VL, Pillatzki A, Gauger P, Schmitt BJ, Koster LG, Killian ML, Yoon KJ. Emergence of Porcine epidemic diarrhea virus in the United States: clinical signs, lesions, and viral genomic sequences. J Vet Diagn Invest 2013, 25, 649-654.

14. Takahashi K, Okada K, Ohshima K. An outbreak of swine diarrhea of a new-type associated with coronaviruslike particles in Japan. Nihon Juigaku Zasshi 1983, 45, 829-832.

15. Tamura K, Dudley J, Nei M, Kumar S. MEGA4: Molecular Evolutionary Genetics Analysis (MEGA) software version 4.0. Mol Biol Evol 2007, 24, 1596-1599.

16. Thompson JD, Gibson TJ, Plewniak F, Jeanmougin F, Higgins DG. The CLUSTAL_X windows interface: flexible strategies for multiple sequence alignment aided by quality analysis tools. Nucleic Acids Res 1997, 25, 4876-4882. 